\title{
Minimal Recurrent Configurations of Chip Firing Games and Directed Acyclic Graphs
}

\author{
Matthias Schulz \\ Karlsruhe Institute for Technology, Dep. of Computer Sciences, Karlsruhe, Germany. Email: schul z @ira.uka.de
}

We discuss a very close relation between minimal recurrent configurations of Chip Firing Games and Directed Acyclic Graphs and demonstrate the usefulness of this relation by giving a lower bound for the number of minimal recurrent configurations of the Abelian Sandpile Model as well as a lower bound for the number of firings which are caused by the addition of two recurrent configurations on particular graphs.

Keywords: Chip Firing Games, Sandpile Model, Minimal Recurrent Configurations, DAGs, Addition of Recurrent Configurations

\section{Introduction}

The Abelian Sandpile Model was introduced by Bak, Tang and Wiesenfeld in 1987 [1] as a model to explain $\frac{1}{f}$ noise. We assign each point of a $n \times n$ grid a number of grains of sand, then taking points which contain at least four grains of sand and let one grain topple to each of the four adjacent points; if a point on the edge of the grid is chosen, grains fall out of the system.

This dynamic is closely related to Chip Firing Games, and Chung and Ellis proposed a variation of Chip Firing Games in 2002 [3] such that the Abelian Sandpile Model can be seen as a special case of this model.

Dhar found many nice properties of so-called recurrent configurations of the Abelian Sandpile Model which are together with a natural operation $\oplus$ an Abelian group, see [5]. These findings can be generalized for Chung and Ellis' Chip Firing Game, as shown in [3].

Recurrent configurations of the Abelian Sandpile Model or Chip Firing Games are characterized by containing enough grains of sand/chips; in this paper we will look at configurations which contain as few chips as possible for a recurrent configuration, and are able to prove a close relation to directed acyclic graphs (DAGs). This relation somewhat resembles the bijection between the set of recurrent configurations of the Sandpile Model and the set of spanning trees with roots at the border of the grid which was shown in [8] and generalized for Chip Firing Games in [3].

These recurrent configurations which we will call minimal play a significant part when considering minimization problems on the set of recurrent configurations, most naturally when minimizing the nuber of firings that occur when relaxing the sum of two recurrent configurations as in [9].

First, we will introduce the basic concepts for Chip Firing Games, before examining the relation between minimal recurrent configurations and a subset of the DAGs on the graph underlying the Chip Firing 


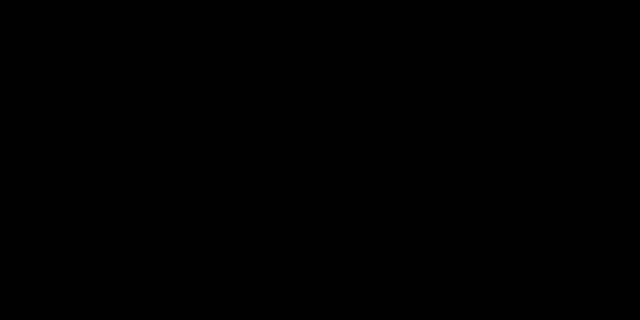

Fig. 1: The vertex in the upper left corner fires, and we get the configuration on the right.

Game. We can use this to prove a lower bound for the number of minimal recurrent configurations of the Abelian Sandpile Model.

Then we will define a dynamic on DAGs which corresponds to the dynamic of the Chip Firing Game. Using this correspondence we will be able to give the infimum of the number of firings that occur when we start the Chip Firing Game on a cylindrical grid with the sum of two recurrent configurations.

\section{Preliminaries}

\subsection{Basic Definitions}

An undirected graph $U=(V \cup S, E)$ is called a $C F G$-graph iff $V$ and $S$ are disjoint, each vertex $s \in S$ is adjacent to exactly one vertex $v \in V$ and there exists a path from each vertex $v \in V$ to a vertex $s \in S$.

A Chip Firing Game (CFG) on a CFG-graph defines a transition rule for configurations $c: V \rightarrow \mathbb{N}_{0}$ where we interpret $c(v)$ as the number of chips the vertex $v$ contains:

If a vertex $v \in V$ contains at least $\operatorname{deg}(v)$ chips, where $\operatorname{deg}(v)$ is the degree of $v$ in the Graph $U$, the vertex $v$ is called critical and can fire, i.e. give one chip to each adjacent vertex and lose $\operatorname{deg}(v)$ chips.

Chips which are given to vertices in $S$ simply vanish from the game. Figure 1 gives an example; black vertices stand for vertices in $S$.

If we start with a configuration $c$ and get configuration $c^{\prime}$ after vertex $v \in V$ has fired, we write $c^{\prime}=\phi_{v}(c)$. We can write

$$
\phi_{v}(c)=c-\operatorname{deg}(v) e_{v}+\sum_{v^{\prime} \mid\left\{v, v^{\prime}\right\} \in E} e_{v^{\prime}}
$$

$e_{v}$ being the configuration given through $\forall u \in V: e_{v}(u)=\delta_{v}(u)$.

A configuration which contains a vertex which is able to fire is called critical; a configuration which is not critical is called stable, and the set of stable configurations is denoted $\mathcal{C}^{U}$.

\subsection{Relaxations of Configurations}

It has been shown (for example in [3]) that we reach a stable configuration after a finite number of firings, no matter which critical configuration we start from. We call the process of these firings the relaxation of $c$. 
For $k \in \mathbb{N}_{0}$ listing the vertices which fired during the first $k$ steps of the relaxation of $c$ is called a firing sequence of $c$ of length $k$.

It is also shown in [3] and [5] that the stable configuration reached does not depend on the sequence of firings - there exists a unique stable configuration $c_{r e l}$ we reach when starting with configuration $c$, and even the number of times a given vertex $v$ fires during the relaxation is unique. The vector $f_{c}$ assigning each vertex the number of times it fires during the relaxation of $c$ is called the firing vector of $c$.

Throughout this paper, when comparing different firing vectors or different configurations, we will use the relation $\leq$ defined through $c \leq d \Longleftrightarrow \forall v \in V: c(v) \leq d(v)$.

\subsection{The Operation $\oplus$ and Recurrent Configurations}

Definition 1 We define the operation $\oplus$ on $\mathcal{C}^{U}$ through

$$
\forall c, d \in \mathcal{C}^{U}: c \oplus d=(c+d)_{\text {rel }}
$$

(The operation + is the usual pointwise addition of functions.)

It is shown in [3] that $\oplus$ is commutative and associative, and also that there exists a subset of stable configurations $\mathcal{R}^{U}$ such that $\left(\mathcal{R}^{U}, \oplus\right)$ is an Abelian group. These configurations are called recurrent configurations.

The structure of the Abelian group $\left(\mathcal{R}^{U}, \oplus\right)$, called the Sandpile Group of the graph $U$, has been the object of research for $U$ being a complete graph or an $n$-wheel in [4] or $U$ being a tree in [7]. Furthermore the geometrical structure of the neutral element of said group has been discussed in [2].

Definition 2 We define $b \in \mathcal{C}^{U}$ as the configuration which assigns to each vertex $v$ the number of vertices in $S$ which are adjacent to $v$. The configuration $b$ is called the burning configuration of $U$.

A generalization of Dhar's Burning Algorithm from [8] gives us the following equivalence:

$\forall c \in \mathcal{C}^{U}: c \in \mathcal{R}^{U} \Longleftrightarrow$ there exists a firing sequence for $c+b$ which contains each vertex exactly once.

(Note that for all $c \in \mathcal{C}^{U}$ the firing sequence of $c+b$ contains each vertex at most once.)

For the rest of this paper, we will say that a sequence $F$ of vertices is a firing sequence for a recurrent configuration $c$ if $F$ is a firing sequence for $c+b$ of length $|V|$.

We are now able to proceed to the actual subject matter of this paper, the set of minimal recurrent configurations.

\section{Minimal Recurrent Configurations and Firing Graphs}

Definition 3 A recurrent configuration $c \in \mathcal{R}^{U}$ is called minimal recurrent if for all vertices $v \in V$ satisfying $c(v)>0$ the configuration $c-e_{v}$ is not recurrent.

The set of all minimal recurrent configurations on $U$ shall be denoted $\mathcal{R}_{\text {min }}^{U}$. In other words $\mathcal{R}_{\text {min }}^{U}$ is the set of minimal elements in $\mathcal{R}^{U}$ with regard to the partial order $\leq$ as defined above.

These minimal recurrent configurations occur naturally when one tries to lower the number of firings that happen during the relaxation of the sum of two recurrent configurations: The function $f: \mathbb{N}_{0}^{V} \rightarrow$ mathbbN $N_{0}^{V}, c \mapsto f_{c}$ is monotonously increasing with regards to $\leq$; this means that $f_{c+d} \leq f_{c^{\prime}+d^{\prime}}$ if $c \leq c^{\prime}$ and $d \leq d^{\prime}$ is true, and we get minimal results for some minimal recurrent configurations $c, d$. 


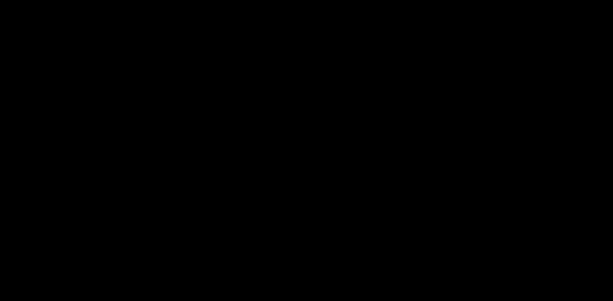

(a) A CFG-Graph $U$ and a recurrent configuration on $U$; the black vertices are the vertices in $S$.

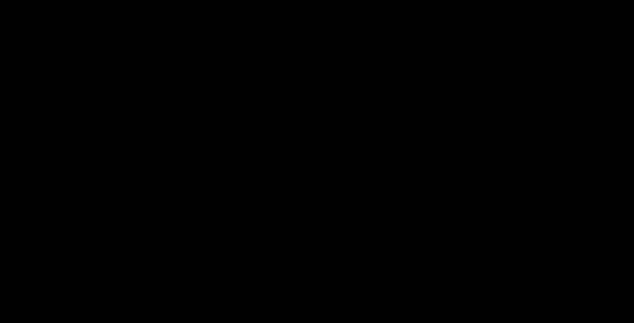

(b) The firing graphs for the firing sequences $(A, B, C, D, E)$ (left) and $(B, A, C, D, E)$ (right).

Fig. 2:

To get a better understanding of minimal recurrent configurations, we use firing graphs, a concept also used by Gajardo and Goles in [6]:

Definition 4 Let $c \in \mathcal{R}^{U}$ be a recurrent configuration and $F=\left(v_{0}, \ldots, v_{|V|-1}\right)$ a firing sequence for $c$.

We define the firing graph $G_{F}=\left(V \cup S, E^{\prime}\right)$ by choosing

$$
E^{\prime}=\left\{\left(v_{i}, v_{j}\right) \mid\left\{v_{i}, v_{j}\right\} \in E \wedge i<j\right\} \cup\{(s, u) \mid s \in S \wedge\{s, u\} \in E\}
$$

We also say that $G_{F}$ is a firing graph for $c$.

Example: The configuration given in Figure 2(a) has the firing sequences $F=(0,1,2,3,4)$ and $F^{\prime}=$ $(1,0,2,3,4)$. The resulting firing graphs $G_{F}$ and $G_{F^{\prime}}$ are shown in Figure 2(b).

Note that $F$ is always a topological ordering of $G_{F}$ restricted to $V$, which implies that $G_{F}$ is always a directed acyclic graph. Note also that for each edge $\{u, v\} \in E$ either $(u, v)$ or $(v, u)$ is an edge in $G$; we will call such acyclic graphs DAGs on $U$ and $S$-DAGs on $U$ if $S$ is the set of sources of $G$.

The set of all $S$-DAGs on $U$ shall be denoted $\mathcal{D}_{S}^{U}$.

Definition 5 As we will be discussing indegrees and outdegrees of vertices in different graphs, we define for a directed graph $G=\left(V \cup S, E^{\prime}\right)$ the functions

$$
\begin{array}{rlrl}
\text { indeg }_{G} & : V \cup S \rightarrow \mathbb{N}_{0}, & v & \mapsto\left|\left\{u \in V \cup S \mid(u, v) \in E^{\prime}\right\}\right| \\
\text { outdeg }_{G}: V \cup S \rightarrow \mathbb{N}_{0}, & v \mapsto\left|\left\{u \in V \cup S \mid(v, u) \in E^{\prime}\right\}\right| .
\end{array}
$$

Note that for all $v \in V$ and all DAGs $G$ on $U$ the equation $\operatorname{indeg}_{G}(v)+\operatorname{outdeg}_{G}(v)=\operatorname{deg}(v)$ is true.

Lemma 1 Let $c \in \mathcal{R}^{U}$ be a recurrent configuration and $G$ be an $S-D A G$ on $U$. Then $G$ is a firing graph of c iff for all vertices $v \in V$ the statement outdeg $g_{G}(v) \leq c(v)$ is true.

Proof: If $G$ is a firing graph of $c$ there exists a firing sequence $F$ of $c$ such that $G=G_{F}$ is true.

It follows that the number of chips fallen to a vertex $v$ before it fires is the number of neighbors firing before it in the firing sequence $F$ plus the numbers of neighbors $v$ has in $S$. These are exactly the vertices from which an edge goes to $v$ in $G$. 
As $v$ has enough chips to fire after the chips of the neighbors mentioned above have fired, it follows $c(v)+\operatorname{indeg}_{G}(v) \geq \operatorname{deg}(v)$, which leads to $c(v) \geq \operatorname{outdeg}_{G}(v)$, which proves one direction.

For the other direction, assume that $\forall v \in V: c(v) \geq \operatorname{outdeg}_{G}(v)$ is true. Let $F=\left(v_{0}, \ldots, v_{|V|-1}\right)$ be a topological ordering of $G$ restricted to $V$.

We show that $F$ is a firing sequence for $c+b$ :

We define for $0 \leq i \leq|V|$ the configuration $c_{i}: V \rightarrow \mathbb{Z}$ as

$$
c_{0}=c+b, \forall i \in\{0, \ldots,|V|-1\}: c_{i+1}=\phi_{v_{i}}\left(c_{i}\right)
$$

In other words, $c_{i}$ is the configuration we get after the first $i$ vertices of the sequence have fired.

To show that $F$ is indeed a firing sequence, we have to prove that for all $i$ the inequation $c_{i}\left(v_{i}\right) \geq$ $\operatorname{deg}\left(v_{i}\right)$ is true.

The number of chips $v_{i}$ contains in $c_{i}$ is the sum of the number of chips $v_{i}$ contained in $c$, the number of neighbors $v_{i}$ has in $S$ and the number of neighbors which fired before $v_{i}$ in $F$; in other words

$$
c_{i}\left(v_{i}\right)=c\left(v_{i}\right)+\operatorname{indeg}_{G}\left(v_{i}\right) \geq \operatorname{outdeg}_{G}\left(v_{i}\right)+\operatorname{indeg}_{G}\left(v_{i}\right)=\operatorname{deg}\left(v_{i}\right)
$$

This completes the proof.

Note that we didn't use the fact that $c$ has to be a recurrent configuration for the second part of the proof; indeed, we have shown we can find a firing sequence for $c+b$ comprising all vertices of $V$ exactly once if $\forall v \in V: c(v) \geq \operatorname{outdeg}_{G}(v)$ is true.

This means that all configurations $c \in \mathcal{C}$ satisfying $\forall v \in V: c(v) \geq \operatorname{outdeg}_{G}(v)$ are recurrent, a fact we state in the following lemma:

Lemma 2 Let $G$ be an $S$-DAG on $U$ and $c \in \mathcal{C}^{U}$ a configuration satisfying $\forall v \in V: c(v) \geq$ outdeg $_{G}(v)$. Then $c$ is a recurrent configuration and $G$ is a firing graph of $c$.

As the sources of $G$ are exactly the vertices in $S$ this means that for all $v \in V$ the inequation $\operatorname{outdeg}_{G}(v) \leq \operatorname{deg}(v)-1$ holds. Therefore such a configuration $c$ always exists in $\mathcal{C}$.

We also can use the firing graphs to prove a lower bound for the number of chips a recurrent configuration contains:

Corollary 1 We define $E_{V} \subseteq E$ as the set of all edges in $U$ incident to two vertices in $V$. Then the following inequation holds:

$$
\forall c \in \mathcal{R}^{U}: \sum_{v \in V} c(v) \geq\left|E_{V}\right|
$$

Proof: Let $c \in \mathcal{R}^{U}$ be a recurrent configuration and $G=\left(V \cup S, E^{\prime}\right)$ be a firing graph of $c$. Using Lemma 2, we get

$$
\forall c \in \mathcal{R}^{U}: \sum_{v \in V} c(v) \geq \sum_{v \in V} \operatorname{outdeg}_{G}(v)
$$

Therefore $c$ contains at least as many chips as there are edges in $G$ starting from a vertex $v \in V$. As each edge $\{u, v\} \in E_{V}$ satisfies $(u, v) \in E^{\prime} \vee(v, u) \in E^{\prime}$ and no edge in $G$ goes from a vertex $v \in V$ to a vertex $s \in S$, we get $\sum_{v \in V}$ outdeg $_{G}(v)=\left|E_{V}\right|$ which proves the claim. 
We know that in a recurrent configuration $c$ with firing graph $G$ each vertex contains at least $\operatorname{outdeg}_{G}(v)$ chips. Looking at configurations where each vertex $v$ contains exactly $\operatorname{outdeg}_{G}(v)$ chips leads us to the following theorem:

Theorem 1 A configuration $c \in \mathcal{C}^{U}$ is minimal recurrent iff there is an $S$-DAG $G$ such that $\forall v \in V$ : $c(v)=\operatorname{outdeg}_{G}(v)$ is true.

Proof: If an $S$-DAG $G$ exists such that $\forall v \in V: c(v)=\operatorname{outdeg}_{G}(v)$ is true, Lemma2 2 tells us that $c$ is a recurrent configuration and $G$ is a firing graph of $c$.

Looking at the proof of Corollary 1 we also get $\sum_{v \in V} c(v)=\sum_{v \in V} \operatorname{outdeg}_{G}(v)=\left|E_{V}\right|$, which is the smallest number of chips a recurrent configuration on $U$ can contain.

For all vertices $v \in V$ the configuration $c-e_{v}$ contains fewer than $\left|E_{V}\right|$ chips, so none of these configurations can be recurrent. Therefore $c$ is a minimal recurrent configuration.

Now, let $c$ be a minimal recurrent configuration and $G$ a firing graph of $c$. We know that $\forall v \in V$ : $c(v) \geq \operatorname{outdeg}_{G}(v)$ is true.

We define $c^{\prime} \in \mathcal{R}^{U}$ as the configuration satisfying $\forall v \in V: c^{\prime}(v)=\operatorname{outdeg}_{G}(v)$. We get $c \geq c^{\prime}$; as $c$ is a minimal recurrent configuration, $c^{\prime}$ must be the same configuration as $c$, which completes the proof.

As we found that the $S$-DAG $G$ claimed to exist for minimal recurrent configuration $C$ in Theorem 1 is a firing graph of $c$, we get the following corollary:

Corollary 2 If $c$ is a minimal recurrent configuration and $G$ is a firing graph of $c, \forall v \in V: c(v)=$ outdeg $_{G}(v)$ is true.

We have shown that there exists a relation between $S$-DAGs on $U$ and minimal recurrent configurations on $U$. We now show that we can even find a bijection between the set of minimal recurrent configurations on $U$ and the set of $S$-DAGs on $U$.

To do so, we have to show that no minimal recurrent configuration has more than one firing graph, which is shown in the following theorem:

Theorem 2 A minimal recurrent configuration has only one firing graph.

Proof: Suppose the minimal recurrent configuration $c$ has two different firing graphs $G_{1}=\left(V \cup S, E_{1}\right)$ and $G_{2}=\left(V \cup S, E_{2}\right)$.

Then we get $\forall v \in V:$ outdeg $_{G_{1}}(v)=c(v)=\operatorname{outdeg}_{G_{2}}(v)$ according to Corollary 2

Consider the set $C=\left\{(u, v) \in E_{1} \mid(v, u) \in E_{2}\right\}$ of edges which are in $G_{1}$ but not in $G_{2}$ and the set $V_{C}=\{v \in V \mid \exists u \in V:(u, v) \in C \vee(v, u) \in C\}$ of vertices incident to edges in $C$. Then the graph $G_{C}=\left(V_{C}, C\right)$ is a subgraph of $G_{1}$ and as such a DAG, which means it that it contains a sink $u$ with outdegree zero and a vertex $v$ such that $(v, u) \in C$.

As $u$ is a sink in $G_{C}$, each vertex $u^{\prime}$ satisfying $\left(u, u^{\prime}\right) \in E_{1}$ also satisfies $\left(u, u^{\prime}\right) \in E_{2}$, as the edge $\left(u, u^{\prime}\right)$ would otherwise be contained in $C$.

We also know that $(u, v) \in E_{2}$, as $(v, u) \in C$. Therefore the outdegree of $u$ in $G_{2}$ is at least one higher than the outdegree of $u$ in $G_{1}$, which is a contradiction. Therefore $c$ can have no two different firing graphs. 
Theorem 2 shows that we can easily assign an $S$-DAG $G$ to each minimal recurrent configuration $c$ by choosing $G$ as the unique firing graph of $c$. We now show that this function is a bijection, which we will afterwards use to prove a lower bound on the number of minimal recurrent configurations if the underlying graph $U$ is a grid.

Definition 6 We define $\tau: \mathcal{R}_{\min }^{U} \rightarrow \mathcal{D}_{S}^{U}$ as the function which is defined through

$$
\forall c \in \mathcal{R}_{\min }^{U}: \tau(c) \text { is the firing graph of } c .
$$

We also define the function $\rho: \mathcal{D}_{S}^{U} \rightarrow \mathcal{R}_{\text {min }}^{U}$ through

$$
\forall G \in \mathcal{D}_{S}^{U}: \forall v \in V:(\rho(G))(v)=\operatorname{outdeg}_{G}(v)
$$

Corollary 3 The functions $\tau$ and $\rho$ are inverse functions and therefore bijections.

Proof: Let $c \in \mathcal{R}_{\text {min }}^{U}$ be a minimal recurrent configuration. We know $\forall v \in V: c(v)=\operatorname{outdeg}_{(\tau(c))(v)}=$ $(\rho(\tau(c)))(v)$ according to Corollary 2, which means that $\rho \circ \tau$ is the identity on $\mathcal{R}_{\text {min }}^{U}$.

Let $G \in \mathcal{D}_{S}^{U}$ be an $S$-DAG of $U$. The configuration $\rho(G)$ is a minimal recurrent configuration according to Lemma 1. As $G$ is a firing graph of $\rho(G)$ according to Lemma 1 and $\rho(G)$ has only one firing graph according to Theorem 2, it follows that $\tau(\rho(G))=G$, and therefore $\tau \circ \rho$ is the identity on $\mathcal{D}_{S}^{U}$.

Therefore $\rho=\tau^{-1}$, which means that $\tau$ and $\rho$ are bijections.

We will use this bijection to prove a lower bound for the number of minimal recurrent configurations of the Abelian Sandpile Model:

Lemma 3 Let $n, m \in \mathbb{N}_{+}$be two positive numbers and $U=(V \cup S, E)$ an $n \times m$ grid with the vertices of $S$ connected to the vertices of the borders of the grid, such that each vertex in the corner of the grid is adjacent to two vertices in $S$ and all other vertices on the borders are adjacent to exactly one vertex in $S$.

Then $\left|\mathcal{R}_{\text {min }}^{U}\right| \geq n \cdot 2^{n(m-1)}+m \cdot 2^{m(n-1)}-n \cdot m$.

This means that the number of minimal recurrent configurations grows exponentially with both the height of the grid as well as the width of the grid.

Proof: As there are exactly as many minimal recurrent configurations on $U$ as there are $S$-DAGs on $U$, we will count a subset of $S$-DAGs on $U$ to get our lower bound. We will refer to vertices $v \in V$ via their coordinates in the grid, starting with $(0,0) \in V$ to $(n-1, m-1) \in V$.

For $k \in\{0, \ldots, n-1\}$ we call a directed graph $G=\left(V \cup S, E^{\prime}\right) k$-divided if

$$
\begin{aligned}
\forall(i, j) \in V: i<k \wedge i+1<n & \Rightarrow((i, j),(i+1, j)) \in E^{\prime} \\
i \geq k \wedge i+1<n & \Rightarrow((i+1, j),(i, j)) \in E^{\prime}
\end{aligned}
$$

and $\forall s \in S: \operatorname{outdeg}_{G}(s)=1$.

See Figure 3 for an example.

It is easy to see that a $k$-divided directed graph is always a DAG whose sources are $S$, i.e each $k$-divided graph $G$ is in $\mathcal{D}_{S}^{U_{n, m}}$ :

If $G$ contained a cycle and we started going round the circle with the edge $((i, j),(i+1, j))$ we would eventually need to get back from a vertex with first component $i+1$ to a vertex with first component $i$, i.e. $G$ would also need to contain an edge $((i+1, k),(i, k))$ which contradicts our definition. 


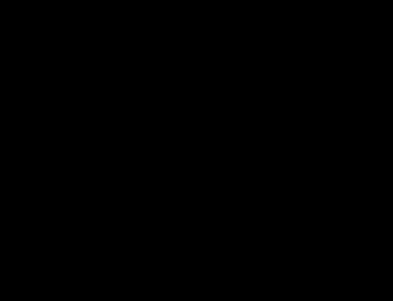

Fig. 3: A 1-divided $3 \times 4$ grid. No matter how the directions of the vertical edges between vertices $v \in V$ are chosen, the resulting graph is a DAG with sources in $S$.

This means that for each edge $\{(i, j),(i, j+1)\} \in E$ we can choose whether to include $((i, j),(i, j+1))$ or $((i, j+1),(i, j))$ in $E^{\prime}$, which gives us $2^{n m-1}$ possibilities to choose a $k$-divided graph for a given $k$.

As we have $n$ different possibilities for $k$, this makes $n 2^{n(m-1)}$ different $S$-DAGs on $U_{n, m}$.

Defining analogously $l$-split directed graphs for $l \in\{0, \ldots, m-1\}$ gives us $m 2^{m(n-1)}$ different $S$ DAGs.

The only graphs counted twice are graphs which are $k$-divided as well as $l$-split for some numbers $k$ and $l$; these are $n m$ different graphs, and we get $n 2^{n(m-1)}+m 2^{m(n-1)}-n m$ different $S$-DAGs on $U_{n, m}$.

Apart from counting minimal recurrent configurations, we can use the relation between DAGs on $U$ and minimal recurrent configurations to find recurrent configurations such that the relaxation of the sum of these configurations takes as few firings as possible.

To do so, we first take a look at DAGs with a set of sources different from $S$ and define a DAG Game on these graphs which corresponds directly with the process of vertices firing in the Chips Firing Game on the same underlying graph..

\section{The DAG Game}

The DAG Game is played with directed acyclic graphs on a CFG-graph $U=(V \cup S, E)$. The simple rule is as follows:

We start with a DAG $G_{1}=\left(V \cup S, E_{1}\right)$ on $U$. In the next step, we take a source $v$ of $G_{1}$ which does not lie in $S$ (if such a source exists) and turn it into a sink by switching the directions of all edges incident to $v$.

In other words the resulting graph $G_{2}=\left(V \cup S, E_{2}\right)$ is defined by

$$
\forall u, u^{\prime} \in V:\left(u, u^{\prime}\right) \in E_{2} \Longleftrightarrow\left(\left(u, u^{\prime}\right) \in E_{1} \wedge u \neq v \neq u^{\prime}\right) \vee\left(\left(u^{\prime}, u\right) \in E_{1} \wedge u^{\prime}=v\right)
$$

See Figure 4 for an example.

If $G_{2}$ contained a cycle $C$ this cycle could not contain the sink $v$; since no edges which are not incident to $v$ have been changed $C$ would also be a cycle in $G_{1}$ which contradicts $G_{1}$ being a DAG. Therefore $G_{2}$ is a DAG, too. 

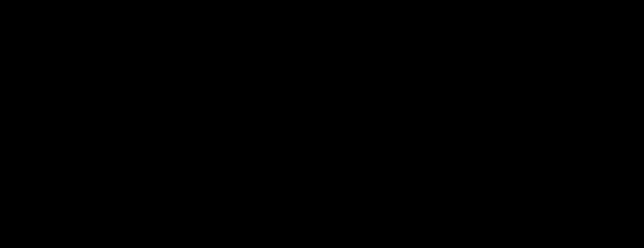

Fig. 4: The central vertex $v$ gets turned into a sink as a step in the DAG Game. The outdegree of all adjacent vertices increases by one.

Let us look at the configurations $c_{G_{1}}$ and $c_{G_{2}}$, again defined by

$$
\forall v \in V: c_{G_{i}}=\operatorname{outdeg}_{G_{i}}(v) .
$$

The only vertices for which $c_{G_{2}}(v) \neq c_{G_{1}}(v)$ is true are $v$ and the vertices adjacent to $v$.

In fact, $c_{G_{1}}(v)=\operatorname{deg}(v)$ and $c_{G_{2}}(v)=0$, while for each neighbor $v^{\prime}$ of $v$ the equation $c_{G_{2}}\left(v^{\prime}\right)=$ $c_{G_{1}}+1$ is true. This means that we get $c_{G_{2}}$ by firing the vertex $v$ in $c_{G_{1}}$.

We use the fact that we can consider the relaxation of a configuration corresponding to a DAG $G$ with sources outside $S$ as repeating steps of the DAG Game starting with $G$ to show that configurations corresponding to two families of DAGs on $U$ relax to minimal recurrent configurations.

Definition 7 A DAG $G$ on $U$ whose set of sinks includes $S$ is called a Sup-S-DAG, denoted $G \in \mathcal{D}_{S+}^{U}$.

A DAG $G$ on $U$ whose set of sinks includes no vertex in $S$ is called a Not- $S$-DAG, denoted $G \in \mathcal{D}_{S-}^{U}$.

We now show that configurations corresponding to Sup- $S$-DAGs or Not- $S$-DAGs always relax to minimal recurrent configurations.

Lemma 4 Let $G$ be a Sup-S-DAG on $U$. Then $c_{G}$ relaxes to a minimal recurrent configuration.

Proof: Consider a sequence $\left(c_{G}=c_{0}, c_{1}, \ldots, c_{k}=\left(c_{G}\right)_{\text {rel }}\right)$ such that for $0 \leq i \leq k-1$ we get $c_{i+1}=\phi_{v_{i}}\left(c_{i}\right)$ for some vertex $v_{i} \in V$.

We show by induction that for each $i \in\{0, \ldots k\}$ there exists a Sup-S-DAG $G_{i}$ on $U$ such that $c_{i}=c_{G_{i}}$ is true, which is obviously the case for $i=0$.

If there exists a Sup-S-DAG $G_{i}$ such that $c_{i}=c_{G_{i}}$ and there exists a vertex $v_{i}$ with $\phi_{v_{i}}\left(c_{i}\right)=c_{i+1}$ this means that outdeg $_{G_{i}}\left(v_{i}\right) \geq \operatorname{deg}\left(v_{i}\right)$.

Since outdeg $G_{i} \leq \operatorname{deg}\left(v_{i}\right)$ this means outdeg $G_{i}\left(v_{i}\right)=\operatorname{deg}\left(v_{i}\right)$ and $v_{i}$ is a source of $G_{i}$. Turning $v_{i}$ into a sink as described above then gives us $G_{i+1}$ such that $c_{G_{i+1}}=c_{i+1}$. All vertices in $S$ still are sources in $G_{i+1}$.

The last DAG $G_{k}$ has no sources outside $S$ as $c_{G_{k}}$ is stable. This means $G_{k}$ is an $S$-DAG and $c_{k}=$ $\left(c_{G}\right)_{r e l} \in \mathcal{R}_{\text {min }}^{U}$.

Lemma 5 Let $G=\left(V \cup S, E^{\prime}\right)$ be a Not-S-DAG on $U$. Then $c_{G}$ relaxes to a minimal recurrent configuration. 
Proof: First, we show that each vertex $v \in V$ fires at least once during the relaxation of $c_{G}$ :

We define the function $p: V \rightarrow \mathbb{N}_{0}$ such that for all $v \in V p(v)$ is the length of the longest path from a source $v^{\prime}$ of $G$ to $v$, formally:

$v \mapsto \max \left\{k \in \mathbb{N}_{0} \mid \exists v^{\prime} \in V: v^{\prime}\right.$ is a source in $G$ and there exists a path from $v^{\prime}$ to $v$ in $G$ of length $\left.k\right\}$.

Instead of looking at the CFG dynamics for the configuration, we consider the corresponding DAG Game dynamics for $G$.

Assume that there exists a vertex $v \in V$ which does not turn into a sink during the relaxation of $c_{G}$ and let $v$ be a vertex with this property for which $p(v)$ is minimal.

Since for all $v^{\prime} \in V$ satisfying $\left(v^{\prime}, v\right) \in E^{\prime}$ the value $p\left(v^{\prime}\right)$ is less than $p(v)$ (as $p(v)=\max \left\{p\left(v^{\prime}\right) \mid\right.$ $\left.\left.\left(v^{\prime}, v\right) \in E^{\prime}\right\}+1\right)$ this means all these vertices $v^{\prime}$ get turned into sinks during the DAG Game. This implies that each edge $\left(v^{\prime}, v\right)$ gets turned into the edge $\left(v, v^{\prime}\right)$.

No vertex $u^{\prime}$ with $\left(v, u^{\prime}\right) \in E^{\prime}$ can become a source as the edge $\left(v, u^{\prime}\right)$ never gets turned to $\left(u^{\prime}, v\right)$ when $v$ cannot turn into a sink. This means that after all vertices $v^{\prime}$ with $\left(v^{\prime}, v\right)$ have been turned into sinks, there is an edge from $v$ to each adjacent vertex $u$.

This means $v$ is a source and can fire in the corresponding configuration, contradicting our assumption.

After each vertex $v \in V$ with an adjacent vertex $s \in S$ has been turned into a sink, all vertices $s \in S$ have become sources, and we get a Sup- $S$-DAG $G^{\prime}$ whose corresponding configuration $c_{G^{\prime}}$ relaxes to a minimal recurrent configuration as shown in Lemma 4

The nice thing about these lemmas is the fact that one gets a Sup-S-DAG if one switches the direction of each edge in a Not- $S$-DAG and vice versa, while the result of the relaxation always is a minimal recurrent configuration.

These property is quite helpful when considering the minimization of the number of firings during the addition of two recurrent configurations, as will be shown in the following section.

\section{Minimizing and Maximizing Firing Vectors}

In this section we will look at how often a vertex $v \in V$ can fire during the relaxation of a configuration $c_{G}$ with $G$ being a DAG on $U$.

We will use the result to consider the question of how many firings there will be at least when relaxing the sum of two recurrent configurations, a problem discussed by the author in [9] where we were able only to give a heuristic algorithm producing recurrent configurations whose sum causes "few" firings during the relaxation.

We can use Lemma 5 to prove a nice and, as we will see later, very helpful property of the configurations $d_{U}^{\prime}, d_{U} \in \mathcal{R}^{U}$ defined as follows:

Definition 8 For a $C F G$-graph $U=(V \cup S, E)$ we define the configuration $d_{U}^{\prime}: V \rightarrow \mathbb{N}_{0}, v \mapsto \operatorname{deg}(v)$ and the configuration $d_{U} \in \mathcal{R}^{U}$ throughd $_{U}=\left(d_{U}^{\prime}\right)_{\text {rel }}$.

As we will now deal with firing vectors, the following lemma will prove useful:

Lemma 6 Let $c, d: V \rightarrow \mathbb{N}_{0}$ be configurations, not necessarily stable. Then $f_{c+d}=f_{c+d_{r e l}}+f_{d}$.

Proof: Any firing sequence $F$ for $d$ is also a firing sequence for $c+d$, as can be easily verified. 
After $F$ we have gotten from $c+d$ to $c+d_{r e l}$, so we get a firing sequence for $c+d$ by concatenating $F$ and a firing sequence $F^{\prime}$ for $c+d_{r e l}$, which means that $f_{c+d}=f_{c+d_{r e l}}+d_{r e l}$ is true.

The configuration $d_{U}$ has a nice property concerning minimal recurrent configurations, as it is very easy to find two minimal recurrent configurations $c$ and $d$ whose relaxed sum is $d_{U}$; also, we can find a close relation between the firing vector of $c+d$ and the firing vector of the configuration $d_{U}^{\prime}-c$, and that in fact the firing vector of $c+d$ gets minimal when the firing vector of $d_{U}^{\prime}-c$ gets maximal.

Lemma 7 Let $G=\left(V \cup S, E^{\prime}\right) \in \mathcal{D}_{S}^{U}$ be an $S$-DAG. Then there exists a Not-S-DAG $G^{\prime} \in \mathcal{D}_{S-}^{U}$ such that $c_{G}+c_{G^{\prime}}=d_{U}^{\prime}$.

Proof: We get $G^{\prime}=\left(V \cup S, E^{\prime \prime}\right)$ by replacing each edge $(u, v) \in E^{\prime}$ through the edge $(v, u)$, formally $E^{\prime \prime}=\left\{(u, v) \mid(v, u) \in E^{\prime}\right\}$.

For all $v \in V$ each vertex $u$ adjacent to $v$ either satisfies $(v, u) \in E^{\prime}$ or $(v, u) \in E^{\prime \prime}$, meaning that outdeg $_{G}(v)+$ outdeg $_{G^{\prime}}(v)=\operatorname{deg}(v)$.

Since all vertices $s \in S$ are sources in $G$, they are sinks in $G^{\prime}$, which completes the proof.

Theorem 3 Let $c \in \mathcal{R}_{\text {min }}^{U}$ be a minimal recurrent configuration and $c^{\prime} \in \mathcal{R}_{\text {min }}^{U}$ be the recurrent configuration satisfying $c \oplus c^{\prime}=d_{U}$. Then the following is true:

(i) $c^{\prime}=\left(d_{U}^{\prime}-c\right)_{\text {rel }}$

(ii) $c^{\prime}$ is a minimal recurrent configuration.

(iii) $f_{c+c^{\prime}}=f_{d_{U}^{\prime}}-f_{d_{U}^{\prime}-c}$

Proof:

(i) Let $G$ be the firing graph for $c$; then $G \in \mathcal{D}_{S}^{U}$.

We turn the direction of each edge of $G$ to get the graph $G^{\prime}$ as in Lemma 7 , and get $c_{G}+c_{G^{\prime}}=$ $d_{U}^{\prime} \Rightarrow c_{G^{\prime}}=d_{U}^{\prime}-c_{G}=d_{U}^{\prime}-c$.

Since $G^{\prime} \in \mathcal{D}_{S-}^{U}$ we get $\left(d_{U}^{\prime}-c\right)_{\text {rel }} \in \mathcal{R}_{\text {min }}^{U}$ from Lemma 5

Therefore $d_{U}=\left(c+\left(d_{U}^{\prime}-c\right)\right)_{\text {rel }}=\left(c+\left(d_{U}^{\prime}-c\right)_{\text {rel }}\right)=c \oplus\left(d_{U}^{\prime}-c\right)_{\text {rel }}$.

As $c^{\prime}$ is unique, we get $c^{\prime}=\left(d_{U}^{\prime}-c\right)_{r e l}$.

(ii) This was shown in the proof to item (i).

(iii) This follows directly from item (i) and Lemma 6 .

We can use Theorem 3 to find minimal recurrent configurations whose sum causes as few as possible firings and relaxes to $d_{U}$ : All we have to do is maximize the number of firings during the relaxation of $d_{U}^{\prime}-c$, which we will do presently.

To minimize the number of firings during the relaxation of the sum of two recurrent configurations one must analyze the pairs of recurrent configurations whose relaxed sum is the configuration $m_{U}$ defined by $\forall v \in V: m_{U}(v)=\operatorname{deg}(v)-1$. This means that we can find the minimal number of firings during the relaxation of the addition of two recurrent configurations if $d_{U}=m_{U}$ is true for the graph $U$. We will give a natural example. 
Lemma 8 For each vertex $v \in V$ and each vertex $s \in S$ let $p(v, s)$ be the length of the shortest path from $v$ to $s$ and $\pi(v)=\min \{p(v, s) \mid s \in S\}$ be the length of the shortest path from $v$ to a vertex in $S$.

Let $G$ be a DAG on $U$ and $c_{G}$ be the corresponding configuration. Then each vertex $v$ fires at most $\pi(v)$ times during the relaxation of $c_{G}$.

Proof: Assume there is a vertex $v \in V$ which fires more than $\pi(v)$ times during the relaxaton of $c_{G}$; let $v$ be a vertex with this property such that $\pi(v)$ is minimal.

Let $v^{\prime} \in V \cup S$ be a vertex adjacent to $v$ with $\pi\left(v^{\prime}\right)=\pi(v)-1$; such a vertex exists on the shortest path from $v$ to a vertex in $S$.

We consider the DAG Game corresponding to the relaxation of $c_{G}$ and discuss the edge between $v$ and $v^{\prime}$. As $\pi\left(v^{\prime}\right)<\pi(v)$ it follows that $v^{\prime}$ fires at most $\pi\left(v^{\prime}\right)=\pi(v)-1$ times; this means that we have at most $\pi(v)-1$ changes from the edge $\left(v^{\prime}, v\right)$ to $\left(v, v^{\prime}\right)$ during the DAG Game.

We also know that $v$ fires at least $\pi(v)+1$ times; this means that the edge $\left(v, v^{\prime}\right)$ changes at least $\pi(v)+1$ times to $\left(v^{\prime}, v\right)$ during the DAG Game. This means that $\left(v, v^{\prime}\right)$ changes at least two times more often to $\left(v^{\prime}, v\right)$ than vice versa. This is impossible, which proves the claim.

Lemma 9 For each $v \in V \pi(v) \in \mathbb{N}_{0}$ shall be defined as in Lemma 8 . We define a sequence $\left(v_{0}, \ldots, v_{|V \cup S|-1}\right)$ of all vertices in $V \cup S$ such that $\forall i, j \in\{0, \ldots,|V \cup S|-1\}: i<j \Rightarrow \pi\left(v_{i}\right)>\pi\left(v_{j}\right)$. The $D A G G=\left(V \cup S, E^{\prime}\right)$ defined by $\left(v_{i}, v_{j}\right) \in E^{\prime} \Longleftrightarrow\left\{v_{i}, v_{j}\right\} \in E \wedge i<j$ satisfies the following: Each vertex $v \in V$ fires exactly $\pi(v)$ times during the relaxation of $c_{G}$.

Proof: Assume the claim is false. Let $k$ be the smallest number such that there exists a vertex $v \in V$ satisfying $\pi(v)>k$ and $v$ fires exactly $k$ times during the relaxation of $c_{G}$.

Let $i$ be the smallest number such that $\pi\left(v_{i}\right)>k$ and $v_{i}$ fires exactly $k$ times during the relaxation of $c_{G}$. If $\left(v_{i}, v_{j}\right) \in E^{\prime}$ we know that $\pi\left(v_{j}\right) \geq k$ and $v_{j}$ fires at least $k$ times during the relaxation of $c_{G}$ since $k$ is minimal. We also know all vertices $v_{j}$ with $j<i$ fire at least $k+1$ times since $\pi\left(v_{j}\right) \geq \pi\left(v_{i}\right)>k$ in these cases and $i$ is minimal.

After all vertices $v_{j}$ with $\left(v_{j}, v_{i}\right) \in E^{\prime}$ have fired $k+1$ times and all vertices $v_{j}$ with $\left(v_{i}, v_{j}\right) \in E^{\prime}$ have fired $k$ times and $v_{i}$ has fired $k$ times, $v_{i}$ has lost $k \cdot \operatorname{deg}\left(v_{i}\right)$ chips and gained $(k+1) \cdot \operatorname{indeg}_{G}\left(v_{i}\right)+k$. $\operatorname{outdeg}_{G}\left(v_{i}\right)=k \cdot \operatorname{deg}\left(v_{i}\right)+\operatorname{indeg}_{G}\left(v_{i}\right)$ chips.

Therefore $v_{i}$ contains at this moment $c_{G}\left(v_{i}\right)+\operatorname{indeg}_{G}\left(v_{i}\right)=\operatorname{deg}\left(v_{i}\right)$ chips and can fire a $k+1$ st time, which contradicts the definitions for $k$ and $v_{i}$. This proves the claim.

Note that we can get a Not- $S$-DAG $G$ as described in Lemma 9 by turning the directions of all edges of the firing graph $G^{\prime}$ given by the firing sequence $\left(v_{|V|-1}, v_{|V|-2}, \ldots, v_{0}\right)$ which starts with vertices adjacent to vertices in $S$.

We now use these DAGs to minimize the number of firings during the relaxation of two recurrent configurations.

Theorem 4 Let $_{U} \in \mathcal{R}^{U}$ be the configuration defined by $\forall v \in V: m_{U}(v)=\operatorname{deg}(v)-1$. Let $c, c^{\prime} \in \mathcal{R}^{U}$ be two recurrent configurations. The DAGs $G$ and $G^{\prime}$ are defined as above.

(i) Let $c^{\prime \prime} \in \mathcal{R}_{\text {min }}^{U}$ be a minimal recurrent configuration satisfying $c^{\prime \prime} \leq c^{\prime}$. Then $f_{c^{\prime \prime}+c^{\prime}} \leq f_{c+c^{\prime}}$.

(ii) We define $e=m_{U}-\left(c \oplus c^{\prime}\right)$. Then $c \oplus\left(c^{\prime} \oplus e\right)=m$ and $f_{c+\left(c^{\prime} \oplus e\right)} \leq f_{c+c^{\prime}}$.

(iii) If $d_{U}=m_{U}$ then $f_{\left(c_{G}\right)_{r e l}+c_{G^{\prime}}} \leq f_{c+c^{\prime}}$ is true. 


\section{Proof:}

(i) This follows directly from the fact that each firing sequence for $c^{\prime \prime}+c^{\prime}$ is a firing sequence for $c+c^{\prime}$ which possibly can be continued.

(ii) $f_{c+\left(c^{\prime} \oplus e\right)}+f_{c^{\prime}+e}=f_{c+c^{\prime}+e}=f_{c+c^{\prime}}+f_{\left(c \oplus c^{\prime}\right)+e}=f_{c+c^{\prime}}$ according to Lemma $6 .\left(f_{\left(c+c^{\prime}\right)+e}=0\right.$ since $\left(c \oplus c^{\prime}\right)+e=m$ is stable.) This proves the claim.

(iii) Items (ii) and (ii) show that there exist minimal recurrent configurations $c_{1}, c_{2} \in \mathcal{R}_{\text {min }}^{U}$ such that $c_{1} \oplus c_{2}=d_{U}$ and $f_{c_{1}+c_{2}} \leq f_{c+c^{\prime}}$ is true.

We know $f_{c_{1}+c_{2}}=f_{d_{U}^{\prime}}-f_{d_{U}^{\prime}-c_{1}}$ from Lemma 3. We also know there exists a DAG $G^{\prime \prime}$ such that $d_{U}^{\prime}-c_{1}=c_{G^{\prime \prime}}$ is true and that each vertex $v \in \nabla$ fires at most $\pi(v)$ times during the relaxation of $c_{G^{\prime \prime}}$. Therefore $f_{c_{G^{\prime \prime}}} \leq f_{c_{G}}$ and $f_{\left(c_{G}\right)_{r e l}+c_{G^{\prime}}}=f_{d_{U}^{\prime}}-f_{c_{G}^{\prime}} \leq f_{d_{U}^{\prime}}-f_{c_{G^{\prime \prime}}}=f_{c_{1}+c_{2}}$ follows.

While there is no algorithm known which produces recurrent configurations such that the sum of these configurations produces a minimal number of firings during the relaxation for the usual Abelian Sandpile Model, the relation between minimal recurrent configurations and $S$-DAGs has given us an easy way to find such configurations when the graph has a special property.

A nice example for a graph $U$ satisfying $d_{U}=m_{U}$ is a cylindrical grid of even height with the vertices of $S$ being above the uppermost and below the lowermost columns of the grid.

If the grid induced by $V$ is a $n \times m$ cylindrical grid and $m$ is even, we can compute that the relaxation of two recurrent configurations leads to at least $n m\left(\frac{m^{2}}{12}-\frac{1}{3}\right)$ firings.

\section{Results}

We have shown that there exists a close relation between DAGs on $U$ and minimal recurrent configurations (minimal with respect to the pointwise $\leq$ ) of the CFG played on $U$, which we used to get a lower bound for the number of minimal recurrent configurations of the sandpile model. Of course, this lower bound could still be improved.

We also found out that graphs corresponding to DAGs $G$ such that either all vertices in $S$ or no vertices in $S$ are sources of $G$ relax to minimal recurrent configurations, which made it easy to show that for each minimal recurrent configuration $c$ the recurrent configuration $c^{\prime}$ such that $c \oplus c^{\prime}=d_{U}$ is minimal recurrent itself.

We could also give a formula for the firing vector $f_{c+c^{\prime}}$ and find the DAG $G$ such that for $c=c_{G}$ the firing vector $f_{c+c^{\prime}}$ becomes minimal. This result was used to give the minimal number of firings that occur when the sum of two recurrent configurations on a cylindrical grid gets relaxed.

These results show that the correspondence between minimal recurrent configurations and DAGs is quite helpful for analyzing recurrent configurations of Chip Firing Games. Future work could try to use this correspondence to find pairs of minimal recurrent configurations whose sum leads to as few firings as possible for underlying graphs not satisfying the condition given in Theorem 4.

Also looking at configurations $c_{G}$ where $G$ is a directed but not acyclic graph might give new insights into the structure of recurrent configurations and configurations "nearly" being recurrent. 


\section{References}

[1] P. Bak, C. Tang, and K. Wiesenfeld, Self-organized criticality: An explanation of the 1/f noise, Phys. Rev. Lett. 59 (1987), 381-384.

[2] Y. Le Borgne and D. Rossin, On the identity of the sandpile group, Discrete Math. 256 (2002), no. 3, $775-790$.

[3] F. Chung and R. Ellis, A chip-firing game and dirichlet eigenvalues, Discrete Mathematics 257 (2002), 341-355.

[4] Robert Cori and Dominique Rossin, On the sandpile group of a graph, European Journal of Combinatorics 21 (2000), 447-459.

[5] D. Dhar, P. Ruelle, S. Sen, and D. N. Verma, Algebraic aspects of abelian sandpile models, J.PHYS.A 28 (1995), 805.

[6] A. Gajardo and E. Goles, Crossing information in two-dimensional sandpiles, Theoretical Computer Science 369 (2006), no. 1-3, 463 - 469.

[7] L. Levine, The sandpile group of a tree, Eur. J. Comb. 30 (2009), no. 4, 1026-1035.

[8] S. N. Majumdar and D. Dhar, Equivalence between the abelian sandpile model and the $q \longrightarrow 0$ limit of the potts model, Physica A: Statistical and Theoretical Physics 185 (1992), 129-145.

[9] M. Schulz, On the addition of recurrent configurations of the sandpile-model, Cellular Automata (H. Umeo, S. Morishita, K. Nishinari, T. Komatsuzaki, and S. Bandini, eds.), Springer Berlin/Heidelberg, 2008, pp. 236-243. 